\title{
One-loop running of dimension-six Higgs-neutrino operators and implications of a large neutrino dipole moment
}

\author{
Mikael Chala ${ }^{a}$ and Arsenii Titov ${ }^{b}$ \\ ${ }^{a}$ CAFPE and Departamento de Física Teórica y del Cosmos, Universidad de Granada, \\ Campus de Fuentenueva, Granada E-18071, Spain \\ ${ }^{b}$ Dipartimento di Fisica e Astronomia "G. Galilei", Università degli Studi di Padova and \\ INFN, Sezione di Padova, Via Francesco Marzolo 8, Padova I-35131, Italy \\ E-mail: mikael.chala@ugr.es, arsenii.titov@pd.infn.it
}

\begin{abstract}
We compute the one-loop running of the dimension-six CP-even Higgs operators in the Standard Model effective field theory involving the right-handed component of the would-be Dirac neutrinos. Then, on the basis of naturalness arguments, for some operators we obtain bounds that surpass direct constraints by orders of magnitude. We also discuss the implications of a large Dirac neutrino magnetic dipole moment. In particular, we demonstrate that a neutrino magnetic moment explaining the recent XENON1T excess induces Higgs and $Z$ invisible decays with branching ratios in the range $\left[10^{-18}, 10^{-12}\right]$. These numbers are unfortunately beyond the reach of current and near future facilities.
\end{abstract}

Keywords: Beyond Standard Model, Higgs Physics, Neutrino Physics

ARXIV EPRINT: 2006.14596 


\section{Contents}

1 Introduction 1

2 The lepton number conserving Standard Model effective field theory 2

3 Computation of the divergences $\quad 5$

4 Anomalous dimensions $\quad 9$

5 Some phenomenological implications 10

6 Conclusions 11

A Cross-checks $\quad \mathbf{1 2}$

$\begin{array}{lll}\text { A.1 } & \bar{N} N \rightarrow B & 13\end{array}$

A.2 $\overline{\nu_{L}} N \rightarrow H_{0} \quad 14$

A.3 $\overline{\nu_{L}} N \rightarrow B H_{0} \quad 14$

A.4 $\overline{e_{L}} N \rightarrow W^{3} H^{+} \quad 14$

A.5 $\bar{N} N \rightarrow H_{0}^{*} H_{0} \quad 15$

A.6 $\overline{\nu_{L}} N H_{0}^{*} \rightarrow H_{0}^{*} H_{0} \quad 16$

$\begin{array}{lll}\text { A.7 } & \bar{N} e_{R} \rightarrow H^{-} H_{0}^{*} & 18\end{array}$

\section{Introduction}

The Standard Model (SM) effective field theory (EFT) $[1,2]$ is the right tool to describe physics above the electroweak (EW) scale. Its use has been boosted in the last years [3] in light of the null results (modulo a few non-conclusive anomalies [4-6]) in the search for new physics at different facilities, and in particular at the LHC. The necessity of using this framework across a wide range of energies has also triggered the computation of the one-loop renormalisation group equations (RGEs) for the dimension-six operators [7-13]. The RGEs in the theory valid at energies below the EW scale where the top quark, the Higgs and the $W$ and $Z$ gauge bosons are integrated out, usually referred to as LEFT, are also known [14]; as well as the matching between the SMEFT and the LEFT at up to one loop $[15,16]$. Likewise, the desire of connecting the SMEFT to ultraviolet (UV) models has stimulated different works on the matching procedure [17-26]; including more recently the first basis of dimension-six operators suitable for off-shell integration [27].

All the aforementioned works assume that neutrinos are Majorana fermions. Notwithstanding the good motivation for this option - in particular lepton number (LN) is only an approximate symmetry of the renormalisable SM Lagrangian - it should not be forgotten that there is absolutely no experimental evidence that neutrinos are not just Dirac particles 
as all the other SM fermions. There is even theoretical support for this. ${ }^{1}$ However, the SMEFT that includes the right-handed $(\mathrm{RH})$ neutrinos $N$, also known as NSMEFT [31, 32], has been explored to a smaller extent; see refs. [33-41] for phenomenological works. The off-shell basis of the NSMEFT has only recently been worked out in ref. [42], where the NLEFT and the tree-level matching between the two EFTs are also presented. More importantly, only the gauge dependence of the RGEs of only very small set of operators are known [43, 44].

Our aim in this paper is to compute the one-loop RGE matrix of the NSMEFT Higgs operators in full detail and to discuss the phenomenological implications, particularly in light of the recent XENON1T observation of an excess of low-energy electron recoil events [6].

The article is organised as follows. In section 2 we introduce the NSMEFT and discuss the generic structure of the RGEs. In section 3 we thoroughly discuss the matching of the UV divergences onto the EFT. We obtain the corresponding counterterms and derive our main result, namely the $5 \times 5$ anomalous dimension matrix to one loop, in section 4 . In section 5 we discuss some phenomenological implications. In particular, the aforementioned XENON1T anomaly might point out to a large neutrino magnetic dipole moment; we demonstrate that it leads to irreducible Higgs and $Z$ invisible decays and we quantify their magnitude. We conclude in section 6 , while appendix $\mathrm{A}$ is dedicated to different cross-checks of our computation.

\section{The lepton number conserving Standard Model effective field theory}

We denote by $e, u$ and $d$ the RH leptons and quarks; and by $L$ and $Q$ the left-handed (LH) counterparts. The gluon and the EW gauge bosons are named by $G$ and $W, B$, respectively. We represent the Higgs doublet by $H=\left(H^{+}, H_{0}\right)^{T}$, and $\tilde{H}=i \sigma_{2} H^{*}$, with $\sigma_{I}, I=1,2,3$, being the Pauli matrices.

Our conventions for the covariant derivative and for the field strength tensors are

$$
D_{\mu}=\partial_{\mu}-i g_{1} Y B_{\mu}-i g_{2} \frac{\sigma^{I}}{2} W_{\mu}^{I}-i g_{s} \frac{\lambda^{A}}{2} G_{\mu}^{A},
$$

and

$$
\begin{aligned}
B_{\mu \nu} & =\partial_{\mu} B_{\nu}-\partial_{\nu} B_{\mu}, \\
W_{\mu \nu}^{I} & =\partial_{\mu} W_{\nu}^{I}-\partial_{\nu} W_{\mu}^{I}+g_{2} \varepsilon^{I J K} W_{\mu}^{J} W_{\nu}^{K}, \\
G_{\mu \nu}^{A} & =\partial_{\mu} G_{\nu}^{A}-\partial_{\nu} G_{\mu}^{A}+g_{s} f^{A B C} G_{\mu}^{B} G_{\nu}^{C},
\end{aligned}
$$

where $Y$ stands for the hypercharge and $\lambda^{A}, A=1, \ldots, 8$, are the Gell-Mann matrices; while $\epsilon^{I J K}$ and $f^{A B C}$ represent the $\mathrm{SU}(2)_{L}$ and $\mathrm{SU}(3)_{c}$ structure constants.

\footnotetext{
${ }^{1}$ For example, refs. [28, 29] show that the SM with only 3 Majorana neutrinos does satisfy the sharpened version of the weak gravity conjecture by Ooguri and Vafa [30], presumably implying that such SM cannot be consistently embedded into a quantum theory of gravity.
} 


\begin{tabular}{|c|c|c|}
\hline 0-Higgs & 1-Higgs & 2-Higgs \\
\hline $\mathcal{O}_{D N}^{1}=\bar{N} \partial^{2} \not \partial N$ & $\mathcal{O}_{N B}=\bar{L} \sigma^{\mu \nu} N \tilde{H} B_{\mu \nu}, \mathcal{O}_{N W}=\bar{L} \sigma^{\mu \nu} N \sigma_{I} \tilde{H} W_{\mu \nu}^{I}$ & $\mathcal{O}_{H N}=\bar{N} \gamma^{\mu} N\left(H^{\dagger} i D_{\mu} H\right)$ \\
$\mathcal{O}_{D N}^{2}=i \tilde{B}_{\mu \nu}\left(\bar{N} \gamma^{\mu} \partial^{\nu} N\right)$ & $\mathcal{O}_{L N}^{1}=\bar{L} N D^{2} \tilde{H}, \mathcal{O}_{L N}^{2}=\bar{L} \partial_{\mu} N D^{\mu} \tilde{H}$ & $\mathcal{O}_{N N}^{2}=\bar{N} i \not N\left(H^{\dagger} H\right)$ \\
$\mathcal{O}_{D N}^{3}=\partial^{\nu} B_{\mu \nu}\left(\bar{N} \gamma^{\mu} N\right)$ & $\mathcal{O}_{L N}^{3}=i \bar{L} \sigma^{\mu \nu} \partial_{\mu} N D_{\nu} \tilde{H}, \mathcal{O}_{L N}^{4}=\bar{L}\left(\partial^{2} N\right) \tilde{H}$ & $\mathcal{O}_{H N e}=\bar{N} \gamma^{\mu} e\left(\tilde{H}^{\dagger} i D_{\mu} H\right)$ \\
\hline \multicolumn{2}{|c|}{ 3-Higgs: $\mathcal{O}_{L N H}=\bar{L} \tilde{H} N\left(H^{\dagger} H\right)$} \\
\hline
\end{tabular}

Table 1. Relevant CP-even bosonic operators. The h.c. is implied when needed. For example, $\mathcal{O}_{D N}^{1}=\bar{N} \partial^{2} \not \partial N+$ h.c. So all Wilson coefficients are hermitian. The CP-odd operators include $i B_{\mu \nu}\left(\bar{N} \gamma^{\mu} \partial^{\nu} N\right), i \mathcal{O}_{N B}, i \mathcal{O}_{N W}, i \mathcal{O}_{L N}^{1,2,3,4}, i \mathcal{O}_{L N H}, i \mathcal{O}_{H N}$ and $i \mathcal{O}_{H N e}[42]$.

\begin{tabular}{|c|c|c|}
\hline \multirow{3}{*}{ 空 } & \multicolumn{2}{|c|}{$\mathcal{O}_{N N}=\left(\bar{N} \gamma_{\mu} N\right)\left(\bar{N} \gamma^{\mu} N\right)$} \\
\hline & $\mathcal{O}_{e N}=\left(\bar{e} \gamma_{\mu} e\right)\left(\bar{N} \gamma^{\mu} N\right)$ & $\mathcal{O}_{u N}=\left(\bar{u} \gamma_{\mu} u\right)\left(\bar{N} \gamma^{\mu} N\right)$ \\
\hline & $\mathcal{O}_{d N}=\left(\bar{d} \gamma_{\mu} d\right)\left(\bar{N} \gamma^{\mu} N\right)$ & $\mathcal{O}_{d u N e}=\left(\bar{d} \gamma_{\mu} u\right)\left(\bar{N} \gamma^{\mu} e\right)$ \\
\hline LLRR & $\mathcal{O}_{L N}=\left(\bar{L} \gamma_{\mu} L\right)\left(\bar{N} \gamma^{\mu} N\right)$ & $\mathcal{O}_{Q N}=\left(\bar{Q} \gamma_{\mu} Q\right)\left(\bar{N} \gamma^{\mu} N\right)$ \\
\hline \multirow{2}{*}{ 茎 } & $\mathcal{O}_{L N L e}=(\bar{L} N) \epsilon(\bar{L} e)$ & $\mathcal{O}_{L N Q d}=(\bar{L} N) \epsilon(\bar{Q} d)$ \\
\hline & \multicolumn{2}{|c|}{$\mathcal{O}_{L d Q N}=(\bar{L} d) \epsilon(\bar{Q} N)$} \\
\hline LRRL & \multicolumn{2}{|c|}{$\mathcal{O}_{Q u N L}=(\bar{Q} u)(\bar{N} L)$} \\
\hline
\end{tabular}

Table 2. CP-even four-fermion operators. The CP-odd ones carry an extra imaginary unit.

We denote by $N$ the $\mathrm{RH}$ component of the neutrino. The renormalisable Lagrangian of the NSMEFT reads

$$
\begin{aligned}
\mathcal{L}_{4}= & -\frac{1}{4} G_{\mu \nu}^{A} G^{A \mu \nu}-\frac{1}{4} W_{\mu \nu}^{I} W^{I \mu \nu}-\frac{1}{4} B_{\mu \nu} B^{\mu \nu} \\
& +\left(D_{\mu} H\right)^{\dagger}\left(D^{\mu} H\right)+\mu_{H}^{2} H^{\dagger} H-\frac{1}{2} \lambda_{H}\left(H^{\dagger} H\right)^{2} \\
& +i(\bar{Q} \not D Q+\bar{u} \not D u+\bar{d} \not D d+\bar{L} \not D L+\bar{e} \not D e+\bar{N} \not D N) \\
& -\left[\bar{Q} Y_{d} H d+\bar{Q} Y_{u} \tilde{H} u+\bar{L} Y_{e} H e+\bar{L} Y_{N} \tilde{H} N+\text { h.c. }\right] .
\end{aligned}
$$

The dimension-six interactions,

$$
\mathcal{L}_{6}=\frac{1}{\Lambda^{2}} \sum_{i} \alpha_{i} \mathcal{O}_{i}
$$

can be expressed in terms of a basis of effective operators. We choose the latter to consist of the SMEFT operators in ref. [2] (which do not contain $N$ ) plus those in tables 1 and 2. The $\alpha_{i}$ represent Wilson coefficients. As we enforce LN conservation, there are no dimension-five operators.

In this work we are only interested in the CP-even sector of the theory. Therefore, in good approximation we can assume that $Y_{u}=\operatorname{diag}\left(y_{u}, y_{c}, y_{t}\right)$, while $Y_{d}=\operatorname{diag}\left(y_{d}, y_{s}, y_{b}\right)$ and $Y_{e}=\operatorname{diag}\left(y_{e}, y_{\mu}, y_{\tau}\right)$ without loss of generality. 
In good approximation we can also assume that there is no huge fine-tuning between the operators entering into the expression for the neutrino mass, $m_{\nu} \sim Y_{N} v-\alpha_{L N H} v^{3} / \Lambda^{2}$, so in particular $Y_{N}$ can be neglected. ${ }^{2}$ This also implies that lepton flavour is conserved in $\mathcal{L}_{4}$. For simplicity we focus on the regime in which lepton flavour is also conserved in the $N$ sector of $\mathcal{L}_{6}$. As a consequence, the three lepton families factorise (in particular they evolve independently under the RGEs). We can therefore ignore flavour indices for clarity.

The operators in grey in tables 1 and 2 are redundant when evaluated on shell; the redundancies due to algebraic or Fierz identities or ensuing from integration by parts have been removed. We refer to this basis as off-shell or Green basis; see ref. [27] for a Green basis of the sector with no $N$.

The relevant equations of motion of $\mathcal{L}_{4}$ for the fermions read:

$$
\begin{aligned}
i \not D L & =Y_{e} H e+Y_{N} \tilde{H} N, \\
i \not \partial N & =Y_{N}^{\dagger} \tilde{H}^{\dagger} L, \\
i \not D e & =Y_{e}^{\dagger} H^{\dagger} L, \\
i \not D Q & =Y_{u} \tilde{H} u+Y_{d} H d, \\
i \not D u & =Y_{u}^{\dagger} \tilde{H}^{\dagger} Q, \\
i \not D d & =Y_{d}^{\dagger} H^{\dagger} Q ;
\end{aligned}
$$

while for the bosons we have instead:

$$
\begin{aligned}
\left(D^{2} \tilde{H}\right)^{i} & =\mu_{H}^{2} \tilde{H}^{i}-\lambda_{H}\left(H^{\dagger} H\right) \tilde{H}^{i}-\epsilon_{i j} \overline{Q^{j}} Y_{d} d-\bar{u} Y_{u}^{\dagger} Q^{i}-\epsilon_{i j} \overline{L^{j}} Y_{e} e-\bar{N} Y_{N} L^{i}, \\
\partial^{\nu} B_{\nu \mu} & =-\frac{g_{1}}{2}\left(i H^{\dagger} D_{\mu} H+\text { h.c. }\right)-g_{1} Y^{f} \bar{f} \gamma_{\mu} f, \\
D^{\nu} W_{\nu \mu}^{I} & =-\frac{g_{2}}{2}\left(H^{\dagger} i D_{\mu}^{I} H-i D_{\mu}^{I} H^{\dagger} H+\bar{L} \gamma_{\mu} \sigma^{I} L+\bar{Q} \gamma_{\mu} \sigma^{I} Q\right), \\
D^{\nu} G_{\nu \mu}^{A} & =-\frac{g_{s}}{2}\left(\bar{Q} \gamma_{\mu} \lambda^{A} Q+\bar{u} \gamma_{\mu} \lambda^{A} u+\bar{d} \gamma_{d} \lambda^{A} d\right) ;
\end{aligned}
$$

where $f$ runs over all fermions. As a consequence, the following relations hold on shell for the operators in grey in table 1 :

$$
\begin{aligned}
\mathcal{O}_{D N}^{1} & =0+\cdots \\
\mathcal{O}_{D N}^{2} & =-\frac{g_{1}}{2} \mathcal{O}_{H N}+\cdots \\
\mathcal{O}_{D N}^{3} & =-\mathcal{O}_{D N}^{2}+\cdots \\
\mathcal{O}_{L N}^{1} & =\left(\mu_{H}^{2} \bar{L} \tilde{H} N+\text { h.c. }\right)-\lambda_{H} \mathcal{O}_{L N H}+\cdots \\
\mathcal{O}_{L N}^{2} & =-\frac{1}{2} Y_{e} \mathcal{O}_{H N}-\left(\frac{\mu_{H}^{2}}{2} \bar{L} \tilde{H} N+\text { h.c. }\right)+\frac{\lambda_{H}}{2} \mathcal{O}_{L N H}-\frac{g_{1}}{8} \mathcal{O}_{N B}+\frac{g_{2}}{8} \mathcal{O}_{N W}+\cdots, \\
\mathcal{O}_{L N}^{3} & =-\mathcal{O}_{L N}^{2}+\cdots
\end{aligned}
$$

\footnotetext{
${ }^{2}$ Even if, as we show below, $\alpha_{L N H}$ is generated radiatively and therefore $Y_{N} \sim g^{2} v^{2} /\left(16 \pi^{2} \Lambda^{2}\right)$ to keep $m_{\nu}$ small, $Y_{N}$ is of order $\lesssim 10^{-4}$ for $\Lambda=1 \mathrm{TeV}$, and hence much smaller than even the muon Yukawa.
} 


$$
\begin{aligned}
\mathcal{O}_{L N}^{4} & =0+\cdots, \\
\mathcal{O}_{N N}^{2} & =0+\cdots
\end{aligned}
$$

The ellipses represent $Y_{N}$ suppressed operators (which might include CP-odd ones) and/or four-fermions, which we ignore. ${ }^{3}$

Under RG running the Wilson coefficients evolve as

$$
\vec{\beta} \equiv 16 \pi^{2} \mu \frac{d \vec{\alpha}}{d \mu}=\gamma \vec{\alpha}
$$

where $\vec{\alpha}$ is a vector that collects the Wilson coefficients of the EFT basis and $\gamma$ is the so-called anomalous dimension matrix.

Given the previous discussion, we can anticipate the global structure of $\gamma$ :

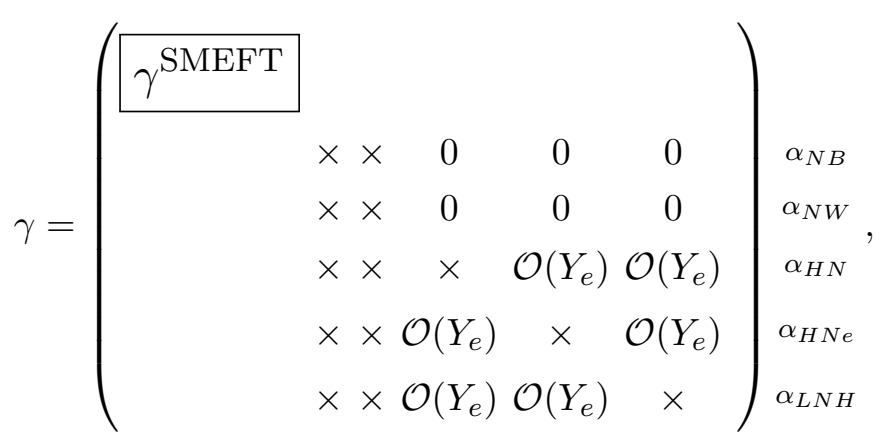

where $\gamma^{\text {SMEFT }}$ stands for the $59 \times 59$ matrix (ignoring flavour indices) accounting for the RG evolution of purely SMEFT operators [10-12]. Because we neglect $Y_{N}, N$ does not interact with any other field at the renormalisable level. Therefore, operators involving $N$ cannot renormalise purely SMEFT operators, and vice versa. This explains the block-diagonal form of the matrix in eq. (2.26).

The almost diagonal structure in the block of $\left\{\mathcal{O}_{H N}, \mathcal{O}_{H N e}, \mathcal{O}_{L N H}\right\}$, only broken by $Y_{e}$, can be explained as follows. Let us define the symmetries $\mathbb{L}_{e}: e \rightarrow \exp \left(i \theta_{e}\right) e, \mathbb{L}_{N}$ : $N \rightarrow \exp \left(i \theta_{N}\right) N$ and $\mathbb{L}_{H}: H \rightarrow \exp \left(i \theta_{H}\right) H$. In the limit $Y_{e} \rightarrow 0, \mathcal{L}_{4}$ is completely invariant under the simultaneous action of $\mathbb{L}_{e}, \mathbb{L}_{N}$ and $\mathbb{L}_{H}$, therefore dimension-four loop corrections to dimension-six operators cannot modify the $e, N$ and Higgs numbers; not even by equations of motion. However, $\mathcal{O}_{H N}, \mathcal{O}_{H N e}$ and $\mathcal{O}_{L N H}$ differ among themselves in at least one of these quantum numbers.

The main result of this paper is the exact one-loop expression for the $5 \times 5$ lower block in $\gamma$, the calculation of which we discuss in detail in the next sections.

\section{Computation of the divergences}

We use the background field method. Each of the gauge bosons is thus split into a background field and a quantum fluctuation that can only appear in loops in Feynman diagrams.

\footnotetext{
${ }^{3}$ While loops of bosonic operators can generate contact interactions, the latter cannot contribute back to bosonic operators via equations of motion and they can therefore be consistently ignored.
} 
We work in the Feynman gauge. The latter is fixed only with respect to the quantum fluctuations, therefore even non-physical quantities such as counterterms are manifestly gauge invariant. Consequently, to order $\mathcal{O}\left(1 / \Lambda^{2}\right)$, any one-loop amplitude (and the divergences themselves) can be unambiguously mapped onto the EFT basis of table 1. Note also that because this Green basis contains operators related by field redefinitions, we can restrict our calculations to (off-shell) one-particle-irreducible amplitudes.

Let us first consider the amplitude for $\bar{N}\left(p_{1}\right) N\left(p_{2}\right) \rightarrow B\left(p_{3}\right)$. Hereafter we work in dimensional regularisation with space-time dimension $d=4-2 \epsilon$ and absorb $1 / \Lambda^{2}$ in the Wilson coefficients in the expressions for amplitudes. Using FeynArts [45] together with FormCalc [46] we obtain the following one-loop divergence to order $\mathcal{O}\left(p^{2}\right)$ :

$$
i \mathcal{M}_{\text {loop }}=\frac{i}{48 \pi^{2} \epsilon} g_{1} \alpha_{H N} \overline{v_{1}}\left(p_{3}^{2} \gamma^{\mu}-p_{3}^{\mu} \not p_{3}\right) P_{R} u_{2} \epsilon_{\mu}^{*} \text {. }
$$

Here and in what follows $v_{1} \equiv v\left(p_{1}\right), u_{2} \equiv u\left(p_{2}\right)$ and $\epsilon_{\mu}^{*} \equiv \epsilon_{\mu}^{*}\left(p_{3}\right)$. The most generic divergence in the EFT depends only on $\mathcal{O}_{D N}^{2}$ and $\mathcal{O}_{D N}^{3}$. It reads

$$
i \mathcal{M}_{\mathrm{div}}=i \bar{v}_{1}\left[\widetilde{\alpha}_{D N}^{3}\left(p_{3}^{\mu} p_{3}-p_{3}^{2} \gamma^{\mu}\right)+2 \widetilde{\alpha}_{D N}^{2}\left(\gamma^{\mu} p_{2} p_{3}-\gamma^{\mu} \not p_{3} p_{2}+p_{3}^{\mu} \not p_{2}-p_{2}^{\mu} \not p_{3}\right)\right] P_{R} u_{2} \epsilon_{\mu}^{*} .
$$

Upon equating $\mathcal{M}_{\text {loop }}$ and $\mathcal{M}_{\text {div }}$, we obtain:

$$
\begin{aligned}
& \widetilde{\alpha}_{D N}^{2}=0 \\
& \widetilde{\alpha}_{D N}^{3}=-\frac{1}{48 \pi^{2} \epsilon} g_{1} \alpha_{H N} .
\end{aligned}
$$

For the amplitude for $\overline{\nu_{L}}\left(p_{1}\right) N\left(p_{2}\right) \rightarrow H_{0}\left(p_{3}\right)$ at $\mathcal{O}\left(p^{2}\right)$ we obtain:

$$
i \mathcal{M}_{\text {loop }}=\frac{i}{32 \pi^{2} \epsilon} \overline{v_{1}}\left[\left(3 g_{1} \alpha_{N B}-9 g_{2} \alpha_{N W}+2 Y_{e} \alpha_{H N e}\right) p_{1}^{2}+Y_{e} \alpha_{H N e} \not p_{1} p_{2}\right] P_{R} u_{2}
$$

and

$$
\begin{aligned}
i \mathcal{M}_{\mathrm{div}}=i \bar{v}_{1}\left[\widetilde{\alpha}_{L N}^{1} p_{1}^{2}\right. & +\left(\widetilde{\alpha}_{L N}^{1}-\widetilde{\alpha}_{L N}^{2}+\widetilde{\alpha}_{L N}^{4}\right) p_{2}^{2} \\
& \left.+\left(2 \widetilde{\alpha}_{L N}^{1}-\widetilde{\alpha}_{L N}^{2}+\widetilde{\alpha}_{L N}^{3}\right) p_{1} p_{2}-\widetilde{\alpha}_{L N}^{3} \not p_{1} \not p_{2}\right] P_{R} u_{2},
\end{aligned}
$$

which implies

$$
\begin{aligned}
& \widetilde{\alpha}_{L N}^{1}=\frac{1}{32 \pi^{2} \epsilon}\left(3 g_{1} \alpha_{N B}-9 g_{2} \alpha_{N W}+2 Y_{e} \alpha_{H N e}\right), \\
& \widetilde{\alpha}_{L N}^{2}=\frac{3}{32 \pi^{2} \epsilon}\left(2 g_{1} \alpha_{N B}-6 g_{2} \alpha_{N W}+Y_{e} \alpha_{H N e}\right), \\
& \widetilde{\alpha}_{L N}^{3}=-\frac{1}{32 \pi^{2} \epsilon} Y_{e} \alpha_{H N e}, \\
& \widetilde{\alpha}_{L N}^{4}=\frac{1}{32 \pi^{2} \epsilon}\left(3 g_{1} \alpha_{N B}-9 g_{2} \alpha_{N W}+Y_{e} \alpha_{H N e}\right) .
\end{aligned}
$$


For the amplitude for $\overline{\nu_{L}}\left(p_{1}\right) N\left(p_{2}\right) \rightarrow B\left(p_{3}\right) H_{0}\left(p_{4}\right)$ at linear order in the external momenta we get:

$$
\begin{aligned}
i \mathcal{M}_{\text {loop }}= & \frac{i}{64 \pi^{2} \epsilon} \overline{v_{1}}\left\{2\left(3 g_{1}^{2} \alpha_{N B}-9 g_{1} g_{2} \alpha_{N W}+2 g_{1} Y_{e} \alpha_{H N e}\right) p_{1}^{\mu}\right. \\
& +2\left[\left(3 g_{2}^{2}-2 g_{1}^{2}+4 Y_{e}^{2}\right) \alpha_{N B}+9 g_{1} g_{2} \alpha_{N W}\right] p_{3}^{\mu}+g_{1} Y_{e} \alpha_{H N e} \gamma^{\mu} \not p_{2} \\
& \left.+\left[\left(g_{1}^{2}-6 g_{2}^{2}-8 Y_{e}^{2}\right) \alpha_{N B}-9 g_{1} g_{2} \alpha_{N W}-2 g_{1} Y_{e} \alpha_{H N e}\right] \gamma^{\mu} \not p_{3}\right\} P_{R} u_{2} \epsilon_{\mu}^{*},
\end{aligned}
$$

as well as

$$
\begin{aligned}
i \mathcal{M}_{\mathrm{div}}=i g_{1} \overline{v_{1}}\left[\widetilde{\alpha}_{L N}^{1} p_{1}^{\mu}\right. & +\left(\widetilde{\alpha}_{L N}^{1}-\frac{1}{2} \widetilde{\alpha}_{L N}^{2}+\frac{1}{2} \widetilde{\alpha}_{L N}^{3}\right) p_{2}^{\mu}+\left(2 \frac{\widetilde{\alpha}_{N B}}{g_{1}}-\frac{1}{2} \widetilde{\alpha}_{L N}^{1}\right) p_{3}^{\mu} \\
& \left.-\frac{1}{2} \widetilde{\alpha}_{L N}^{3} \gamma^{\mu} p_{2}-2 \frac{\widetilde{\alpha}_{N B}}{g_{1}} \gamma^{\mu} \not p_{3}\right] P_{R} u_{2} \epsilon_{\mu}^{*} .
\end{aligned}
$$

Upon equating both quantities, we obtain the same values of $\widetilde{\alpha}_{L N}^{1}, \widetilde{\alpha}_{L N}^{2}$ and $\widetilde{\alpha}_{L N}^{3}$ as before (what provides a strong cross-check of the computation), as well as

$$
\widetilde{\alpha}_{N B}=\frac{1}{128 \pi^{2} \epsilon}\left[\left(6 g_{2}^{2}-g_{1}^{2}+8 Y_{e}^{2}\right) \alpha_{N B}+9 g_{1} g_{2} \alpha_{N W}+2 g_{1} Y_{e} \alpha_{H N e}\right] .
$$

The divergences at one loop and in the EFT for $\overline{e_{L}}\left(p_{1}\right) N\left(p_{2}\right) \rightarrow W^{3}\left(p_{3}\right) H^{+}\left(p_{4}\right)$ at $\mathcal{O}(p)$ read respectively:

$$
\begin{aligned}
i \mathcal{M}_{\text {loop }}=\frac{i}{64 \pi^{2} \epsilon} \overline{v_{1}}\{ & 2 g_{2}\left(9 g_{2} \alpha_{N W}-3 g_{1} \alpha_{N B}-2 Y_{e} \alpha_{H N e}\right) p_{1}^{\mu} \\
& +2\left[3 g_{1} g_{2} \alpha_{N B}+\left(g_{1}^{2}-6 g_{2}^{2}-4 Y_{e}^{2}\right) \alpha_{N W}\right] p_{3}^{\mu} \\
& -g_{2} Y_{e} \alpha_{H N e} \gamma^{\mu} p_{2} \\
& \left.-\left[3 g_{1} g_{2} \alpha_{N B}+\left(2 g_{1}^{2}-3 g_{2}^{2}-8 Y_{e}^{2}\right) \alpha_{N W}-2 g_{2} Y_{e} \alpha_{H N e}\right] \gamma^{\mu} \not p_{3}\right\} P_{R} u_{2} \epsilon_{\mu}^{*},
\end{aligned}
$$

and

$$
\begin{aligned}
i \mathcal{M}_{\mathrm{div}}=i g_{2} \overline{v_{1}}\left[-\widetilde{\alpha}_{L N}^{1} p_{1}^{\mu}\right. & -\left(\widetilde{\alpha}_{L N}^{1}-\frac{1}{2} \widetilde{\alpha}_{L N}^{2}+\frac{1}{2} \widetilde{\alpha}_{L N}^{3}\right) p_{2}^{\mu}+\left(2 \frac{\widetilde{\alpha}_{N W}}{g_{2}}+\frac{1}{2} \widetilde{\alpha}_{L N}^{1}\right) p_{3}^{\mu} \\
+ & \left.\frac{1}{2} \widetilde{\alpha}_{L N}^{3} \gamma^{\mu} \not p_{2}-2 \frac{\widetilde{\alpha}_{N W}}{g_{2}} \gamma^{\mu} \not p_{3}\right] P_{R} u_{2} \epsilon_{\mu}^{*}
\end{aligned}
$$

This cross-checks again $\widetilde{\alpha}_{L N}^{1}, \widetilde{\alpha}_{L N}^{2}$ and $\widetilde{\alpha}_{L N}^{3}$ and also leads to

$$
\widetilde{\alpha}_{N W}=\frac{1}{128 \pi^{2} \epsilon}\left[3 g_{1} g_{2} \alpha_{N B}+\left(2 g_{1}^{2}-3 g_{2}^{2}-8 Y_{e}^{2}\right) \alpha_{N W}-2 g_{2} Y_{e} \alpha_{H N e}\right] .
$$

For $\bar{N}\left(p_{1}\right) N\left(p_{2}\right) \rightarrow H_{0}^{*}\left(p_{3}\right) H_{0}\left(p_{4}\right)$ to $\mathcal{O}(p)$ in the external momenta, we have:

$$
i \mathcal{M}_{\text {loop }}=\frac{i}{32 \pi^{2} \epsilon}\left(g_{1}^{2}+3 g_{2}^{2}\right) \alpha_{H N} \overline{v_{1}}\left(\not p_{1}+\not p_{2}-2 \not p_{3}\right) u_{2},
$$


and

$$
i \mathcal{M}_{\mathrm{div}}=i \overline{v_{1}}\left[\left(\widetilde{\alpha}_{N N}^{2}-\widetilde{\alpha}_{H N}\right) \not p_{1}-\left(\widetilde{\alpha}_{N N}^{2}+\widetilde{\alpha}_{H N}\right) \not p_{2}+2 \widetilde{\alpha}_{H N} \not p_{3}\right] P_{R} u_{2} .
$$

From equating these two amplitudes, we obtain the conditions:

$$
\begin{aligned}
& \widetilde{\alpha}_{N N}^{2}=0 \\
& \widetilde{\alpha}_{H N}=-\frac{1}{32 \pi^{2} \epsilon}\left(g_{1}^{2}+3 g_{2}^{2}\right) \alpha_{H N} .
\end{aligned}
$$

The one-loop divergence for $\overline{\nu_{L}}\left(p_{1}\right) N\left(p_{2}\right) H_{0}^{*}\left(p_{3}\right) \rightarrow H_{0}^{*}\left(p_{4}\right) H_{0}\left(p_{5}\right)$ at zero momentum reads

$$
\begin{aligned}
i \mathcal{M}_{\text {loop }}=\frac{i}{16 \pi^{2} \epsilon} \overline{v_{1}}[ & \left(12 \lambda_{H}-g_{1}^{2}-3 g_{2}^{2}-2 Y_{e}^{2}\right) \alpha_{L N H}-3 g_{1}\left(g_{1}^{2}+g_{2}^{2}\right) \alpha_{N B} \\
& \left.+3 g_{2}\left(g_{1}^{2}+3 g_{2}^{2}+4 Y_{e}^{2}\right) \alpha_{N W}+Y_{e}\left(3 g_{2}^{2}-2 \lambda_{H}-2 Y_{e}^{2}\right) \alpha_{H N e}\right] P_{R} u_{2}
\end{aligned}
$$

while in the EFT at tree level we have

$$
i \mathcal{M}_{\mathrm{div}}=-2 i \widetilde{\alpha}_{L N H} \overline{v_{1}} P_{R} u_{2}
$$

This fixes

$$
\begin{aligned}
\widetilde{\alpha}_{L N H}=\frac{1}{32 \pi^{2} \epsilon}[ & 3 g_{1}\left(g_{1}^{2}+g_{2}^{2}\right) \alpha_{N B}-3 g_{2}\left(g_{1}^{2}+3 g_{2}^{2}+4 Y_{e}^{2}\right) \alpha_{N W} \\
& \left.+Y_{e}\left(2 \lambda_{H}-3 g_{2}^{2}+2 Y_{e}^{2}\right) \alpha_{H N e}+\left(g_{1}^{2}+3 g_{2}^{2}-12 \lambda_{H}+2 Y_{e}^{2}\right) \alpha_{L N H}\right] .
\end{aligned}
$$

Finally, upon computing the divergent part of $\bar{N}\left(p_{1}\right) e_{R}\left(p_{2}\right) \rightarrow H^{-}\left(p_{3}\right) H_{0}^{*}\left(p_{4}\right)$ at order $\mathcal{O}(p)$, we obtain:

$$
i \mathcal{M}_{\text {loop }}=\frac{3 i}{32 \pi^{2} \epsilon}\left[g_{1} Y_{e} \alpha_{N B}-3 g_{2} Y_{e} \alpha_{N W}+\left(g_{1}^{2}-g_{2}^{2}\right) \alpha_{H N e}\right] \overline{v_{1}}\left(\not p_{3}-\not p_{4}\right) P_{R} u_{2}
$$

and

$$
i \mathcal{M}_{\mathrm{div}}=i \widetilde{\alpha}_{H N e} \overline{v_{1}}\left(\not p_{3}-\not p_{4}\right) P_{R} u_{2}
$$

which leads to

$$
\widetilde{\alpha}_{H N e}=\frac{3}{32 \pi^{2} \epsilon}\left[g_{1} Y_{e} \alpha_{N B}-3 g_{2} Y_{e} \alpha_{N W}+\left(g_{1}^{2}-g_{2}^{2}\right) \alpha_{H N e}\right] .
$$

We provide a completely independent cross-check of these results in appendix A. 
To conclude, for the $Z$ factors of the fields we have:

$$
\begin{aligned}
& Z_{H}=1+\frac{1}{32 \pi^{2} \epsilon}\left[g_{1}^{2}+3 g_{2}^{2}-6 \operatorname{Tr}\left(Y_{u}^{2}+Y_{d}^{2}\right)-2 \operatorname{Tr}\left(Y_{e}^{2}\right)\right] \\
& Z_{L}=1-\frac{1}{64 \pi^{2} \epsilon}\left(g_{1}^{2}+3 g_{2}^{2}+2 Y_{e}^{2}\right), \\
& Z_{e}=1-\frac{1}{16 \pi^{2} \epsilon}\left(g_{1}^{2}+Y_{e}^{2}\right) \\
& Z_{B}=1-\frac{41 g_{1}^{2}}{96 \pi^{2} \epsilon} \\
& Z_{W}=1+\frac{19 g_{2}^{2}}{96 \pi^{2} \epsilon} .
\end{aligned}
$$

Note that we use $Y_{e}$ to refer both to a particular entry of the Yukawa matrix and to this matrix itself (when it comes inside the trace).

\section{Anomalous dimensions}

We remove the redundant operators (those in grey in table 1) using the relations in eqs. (2.17)-(2.24). This shifts the Wilson coefficients $\alpha_{N B}, \alpha_{N W}$ and $\alpha_{H N}$ :

$$
\begin{gathered}
\widetilde{\alpha}_{N B} \rightarrow \widetilde{\alpha}_{N B}-\frac{g_{1}}{8}\left(\widetilde{\alpha}_{L N}^{2}-\widetilde{\alpha}_{L N}^{3}\right)=\frac{1}{64 \pi^{2} \epsilon}\left[\left(3 g_{2}^{2}-2 g_{1}^{2}+4 Y_{e}^{2}\right) \alpha_{N B}+9 g_{1} g_{2} \alpha_{N W}\right], \\
\widetilde{\alpha}_{N W} \rightarrow \widetilde{\alpha}_{N W}+\frac{g_{2}}{8}\left(\widetilde{\alpha}_{L N}^{2}-\widetilde{\alpha}_{L N}^{3}\right)=\frac{1}{64 \pi^{2} \epsilon}\left[3 g_{1} g_{2} \alpha_{N B}+\left(g_{1}^{2}-6 g_{2}^{2}-4 Y_{e}^{2}\right) \alpha_{N W}\right], \\
\widetilde{\alpha}_{H N} \rightarrow \widetilde{\alpha}_{H N}-\frac{g_{1}}{2}\left(\widetilde{\alpha}_{D N}^{2}-\widetilde{\alpha}_{D N}^{3}\right)-\frac{Y_{e}}{2}\left(\widetilde{\alpha}_{L N}^{2}-\widetilde{\alpha}_{L N}^{3}\right) \\
=-\frac{1}{96 \pi^{2} \epsilon}\left[9 g_{1} Y_{e} \alpha_{N B}-27 g_{2} Y_{e} \alpha_{N W}+\left(4 g_{1}^{2}+9 g_{2}^{2}\right) \alpha_{H N}+6 Y_{e}^{2} \alpha_{H N e}\right]
\end{gathered}
$$

$\alpha_{H N e}$ and $\alpha_{L N H}$ (accidentally) remain unchanged.

This way, we fully determine the divergent Lagrangian

$$
\mathcal{L}_{\text {div }}=\frac{1}{32 \pi^{2} \Lambda^{2} \epsilon} \overrightarrow{\mathcal{O}}^{T} \cdot \mathcal{C} \cdot \vec{\alpha}
$$

where the vector $\overrightarrow{\mathcal{O}}$ encodes the relevant operators, and the matrix $\mathcal{C}$ contains only SM couplings. We use the latter to fix the counterterms in the NSMEFT Lagrangian

$$
\begin{aligned}
\mathcal{L}_{6} & =\frac{1}{\Lambda^{2}} \vec{\alpha}^{T} \cdot \overrightarrow{\mathcal{O}}+\frac{1}{\Lambda^{2}} \overrightarrow{\mathcal{O}^{T}} \cdot\left(Z_{F} Z-\mathbb{1}\right) \cdot \vec{\alpha} \\
& =\frac{1}{\Lambda^{2}} \vec{\alpha}^{T} \cdot \overrightarrow{\mathcal{O}}+\frac{1}{32 \pi^{2} \Lambda^{2} \epsilon} \overrightarrow{\mathcal{O}^{T}} \cdot\left(K_{F}+K\right) \cdot \vec{\alpha},
\end{aligned}
$$

where $Z_{F}$ contains the wave-function renormalisation factors, ${ }^{4}$ and we have introduced $Z=\mathbb{1}+K /\left(32 \pi^{2} \epsilon\right)$ and $Z_{F}=\mathbb{1}+K_{F} /\left(32 \pi^{2} \epsilon\right)$. We obtain

$$
K=-\left(\mathcal{C}+K_{F}\right) \text {. }
$$

\footnotetext{
${ }^{4}$ Explicitly,

$$
Z_{F}=\operatorname{diag}\left(\sqrt{Z_{L} Z_{H} Z_{B}}, \sqrt{Z_{L} Z_{H} Z_{W}}, Z_{H}, \sqrt{Z_{E}} Z_{H}, \sqrt{Z_{L}}\left(Z_{H}\right)^{3 / 2}\right)
$$
}


Following e.g. ref. [47], it can be seen that the anomalous dimension matrix $\gamma$ is simply given by $K$. Thus, we finally get

$$
\gamma=\left(\begin{array}{ccccc}
\frac{91}{12} g_{1}^{2}-\frac{9}{4} g_{2}^{2}-\frac{3}{2} Y_{e}^{2}+\operatorname{Tr}^{2} & -\frac{9}{2} g_{1} g_{2} & 0 & 0 & 0 \\
-\frac{3}{2} g_{1} g_{2} & -\frac{3}{4} g_{1}^{2}-\frac{11}{12} g_{2}^{2}+\frac{5}{2} Y_{e}^{2}+\operatorname{Tr}^{2} & 0 & 0 & 0 \\
3 g_{1} Y_{e} & -9 g_{2} Y_{e} & \frac{1}{3} g_{1}^{2}+2 \operatorname{Tr}^{2} & 2 Y_{e}^{2} & 0 \\
-3 g_{1} Y_{e} & 9 g_{2} Y_{e} & 0 & -3 g_{1}^{2}+Y_{e}^{2}+2 \operatorname{Tr}^{2} & 0 \\
-3 g_{1}\left(g_{1}^{2}+g_{2}^{2}\right) & 3 g_{2}\left(g_{1}^{2}+3 g_{2}^{2}+4 Y_{e}^{2}\right) & 0 & Y_{e}\left(3 g_{2}^{2}-2 \lambda_{H}-2 Y_{e}^{2}\right) & -\frac{9}{4} g_{1}^{2}-\frac{27}{4} g_{2}^{2}+12 \lambda_{H}-\frac{3}{2} Y_{e}^{2}+3 \operatorname{Tr}^{2}
\end{array}\right) \alpha_{L N H}, \alpha_{N B},
$$

where we have defined

$$
\operatorname{Tr}^{2} \equiv 3 \operatorname{Tr}\left(Y_{u}^{2}+Y_{d}^{2}\right)+\operatorname{Tr}\left(Y_{e}^{2}\right) .
$$

We notice that, as anticipated in section 2, the operators $\mathcal{O}_{H N}, \mathcal{O}_{H N e}$ and $\mathcal{O}_{L N H}$ do not mix in the limit of vanishing $Y_{e}$. Also, these operators, which can be generated at tree level in UV completions of the SM, do not renormalise $\mathcal{O}_{N W}$ and $\mathcal{O}_{N B}$, which can only arise at one loop. We also stress that some entries, e.g. the renormalisation of $\mathcal{O}_{H N}$ by $\mathcal{O}_{N W}$, are enhanced with respect to the naive dimensional analysis estimation by up to an order of magnitude.

\section{Some phenomenological implications}

Among the variety of phenomenological implications, we would like to explore the possibility that the excess of low-energy electron recoil events recently observed by XENON1T [6], which has triggered a lot of attention [48-71], is due to a relatively large neutrino magnetic dipole moment. Following ref. [72] (see also ref. [52]), one can take $\mu_{\nu} \sim 2 \times 10^{-11} \mu_{B}$, where $\mu_{B}$ stands for the Bohr magneton. (This explanation necessarily assumes that the strong astrophysical bounds [73], which are subject to a number of uncontrollable uncertainties, can not be taken at face value.)

The neutrino magnetic moment can also be expressed as [43]

$$
\left|\frac{\mu_{\nu}}{\mu_{B}}\right|=\frac{4 \sqrt{2}}{\mathrm{e}} \frac{m_{e} v}{\Lambda^{2}} \alpha_{N A}(v)
$$

where $\alpha_{N A}=c_{W} \alpha_{N B}+s_{W} \alpha_{N W}, m_{e}$ represents the electron mass and e $=\sqrt{4 \pi \alpha_{\mathrm{QED}}}$ with $\alpha_{\mathrm{QED}}$ the electromagnetic fine-structure constant. The non detection of new particles at the LHC most likely implies that $\alpha_{N A}$ is generated at a scale $\Lambda \gtrsim \mathrm{TeV}$. In what follows we assume two benchmark values of $\Lambda=1 \mathrm{TeV}$ and $100 \mathrm{TeV}$. We obtain

$$
\alpha_{N A} \sim 9 \times 10^{-6}\left(9 \times 10^{-2}\right) \text { for } \Lambda=1 \mathrm{TeV}(100 \mathrm{TeV}) .
$$

Even if this is the only non-vanishing Wilson coefficient at the high scale, running down from $\Lambda=1 \mathrm{TeV}(100 \mathrm{TeV})$ to the EW scale, we obtain:

$$
\begin{aligned}
\left|\alpha_{L N H}(v)\right| & \sim 6 \times 10^{-8}\left(2 \times 10^{-3}\right), \\
\left|\alpha_{H N}(v)\right| & \sim 10^{-9}\left(6 \times 10^{-5}\right), \\
\left|\alpha_{N Z}(v)\right| & \sim 2 \times 10^{-8}\left(7 \times 10^{-4}\right),
\end{aligned}
$$


where $\alpha_{N Z}=c_{W} \alpha_{N W}-s_{W} \alpha_{N B}$. (We have assumed that all neutrinos have similar magnetic moment, so $\alpha_{H N}$ is only suppressed by the tau Yukawa.)

An immediate consequence of this result is that the neutrino masses get a radiative contribution of order $\delta m_{\nu}=\left|\alpha_{L N H}\right| v^{3} /\left(2 \sqrt{2} \Lambda^{2}\right) \sim 3 \times 10^{2} \mathrm{eV}\left(10^{3} \mathrm{eV}\right)$ for the new physics scale $\Lambda=1 \mathrm{TeV}(100 \mathrm{TeV})$. Most of this correction must be cancelled by the bare $Y_{N}$, implying a fine-tuning of order $\mathcal{O}\left(10^{3}-10^{4}\right)$. This observation was already made in ref. [43]. The authors of this article obtain the RGEs of $\alpha_{N B}, \alpha_{N W}$ and $\alpha_{L N H}$ neglecting the Yukawa terms. The equivalent block in our $\gamma$ matches their result up to a factor of 2 in the mixing of $\alpha_{N B}$ and $\alpha_{N W}$ into $\alpha_{L N H}$, which (slightly) weakens the amount of fine-tuning. Unfortunately, we do not find enough details about the computation in ref. [43] to disentangle the root of this discrepancy.

Irrespectively of this tuning, given the aforementioned numbers for the Wilson coefficients and taking into account the three lepton families, we predict the following Higgs and $Z$ decays for $\Lambda=1 \mathrm{TeV}(100 \mathrm{TeV})$ :

$$
\begin{aligned}
\Gamma(h \rightarrow \text { inv }) & =\frac{3 m_{h} v^{4}}{16 \pi \Lambda^{4}} \alpha_{L N H}^{2} \sim 9 \times 10^{-14} \mathrm{MeV}\left(2 \times 10^{-12} \mathrm{MeV}\right), \\
\Gamma(Z \rightarrow \operatorname{inv}) & =\frac{m_{Z}^{3} v^{2}}{8 \pi \Lambda^{4}}\left(\alpha_{H N}^{2}+2 \alpha_{N Z}^{2}\right) \sim 10^{-18} \mathrm{GeV}\left(2 \times 10^{-17} \mathrm{GeV}\right) .
\end{aligned}
$$

The expected Higgs and $Z$ branching ratios are therefore

$$
\begin{aligned}
\mathcal{B}(h \rightarrow \text { inv }) & \sim 2 \times 10^{-14}\left(4 \times 10^{-13}\right), \\
\mathcal{B}(Z \rightarrow \text { inv }) & \sim 5 \times 10^{-19}\left(8 \times 10^{-18}\right),
\end{aligned}
$$

where we have used $\Gamma_{h}^{\text {total }} \approx 4 \mathrm{MeV}$ and $\Gamma_{Z}^{\text {total }} \approx 2.49 \mathrm{GeV}$ [74]. Unfortunately, these numbers are so small that it is not feasible that they will be tested at any current or future facilities [75].

On a different front, from eq. (4.8) it is also clear that $\mathcal{O}_{H N e}$ generates a contribution $\delta m_{\nu}$ to the neutrino mass too. For the tau flavour, requiring $\delta m_{\nu}<1 \mathrm{eV}$, it can be shown that $\alpha_{H N e} \lesssim 2 \times 10^{-6}$ for $\Lambda=1 \mathrm{TeV}$. If $\alpha_{H N e}$ is rather generated at $\Lambda=100 \mathrm{TeV}$, we obtain $\alpha_{H N e} \lesssim 2 \times 10^{-2}$. These bounds surpass by orders of magnitude the best bound that can be set on $\alpha_{H N e}$ using measurements of $W$ branching ratios, which is $\mathcal{O}(1) .{ }^{5}$

\section{Conclusions}

We have computed the RGEs of all dimension-six Higgs operators in the NSMEFT at one loop, thereby extending previous partial computations which did not include all the operators nor the Yukawa dependence. Thus, this work comprises a substantial step forward

\footnotetext{
${ }^{5}$ Note that assuming $\alpha_{N W}=0$,

$$
\Delta \Gamma(W \rightarrow \ell \nu)=\frac{m_{W}^{3} v^{2}}{48 \pi \Lambda^{4}} \alpha_{H N e}^{2}
$$

while experimentally this quantity is bounded to $\Delta \Gamma(W \rightarrow \ell \nu) / \Gamma_{W}^{\text {total }}<2 \times 10^{-3}$ at the 95\% CL [74], with $\Gamma_{W}^{\text {total }} \sim 2.09 \mathrm{GeV}$. Altogether this implies $\left|\alpha_{H N e} / \Lambda^{2}\right| \lesssim 4.5 \mathrm{TeV}^{-2}$.
} 
towards the description of new physics in terms of EFTs in the regime in which neutrinos are Dirac particles.

In our basis, the only operators that do not mix among themselves under running are $\mathcal{O}_{H N}$ and $\mathcal{O}_{L N H}$, while the three operators $\mathcal{O}_{H N}, \mathcal{O}_{H N e}$ and $\mathcal{O}_{L N H}$ renormalise independently in the limit of vanishing Yukawas (even at higher orders).

The operators $\mathcal{O}_{N B}$ and $\mathcal{O}_{N W}$, which together contribute to the neutrino magnetic dipole moment, renormalise $\mathcal{O}_{L N H}$ via gauge interactions; all the others are Yukawa suppressed. With this in mind, we have also analysed the consequences of the recent XENON1T excess [6] being due to an anomalous Dirac neutrino magnetic dipole moment $\mu_{\nu} \sim 2 \times 10^{-11} \mu_{B}$. We observe that:

1. A contribution to the neutrino mass of order $10^{2}-10^{3} \mathrm{eV}$ would be generated, requiring a sensible cancellation between this and the bare mass to account for the tiny observed $m_{\nu} \sim 0.1 \mathrm{eV}$. This was already pointed out in ref. [43]. We however find a small discrepancy with the result in this reference; see section 5 .

2. Irrespectively of whether neutrino masses are tuned, $\mathcal{O}_{L N H}$ would be induced radiatively triggering the Higgs decay to invisible $h \rightarrow$ inv with branching ratio of order $\sim 2 \times 10^{-14}\left(4 \times 10^{-13}\right)$ for $\Lambda=1 \mathrm{TeV}(100 \mathrm{TeV})$.

3. If the dipole moment of the electron and tau neutrinos are equally large, then one also expects a new contribution to the invisible $Z$ decay with branching ratio $5 \times 10^{-19}$ $\left(8 \times 10^{-18}\right)$ for $\Lambda=1 \mathrm{TeV}(100 \mathrm{TeV})$.

Unfortunately, even if the XENON1T excess survives in the long term, these numbers are too small to be explored at current and near future facilities.

On a different note, we have shown that $\mathcal{O}_{H N e}$ also renormalises the neutrino mass term by $\delta m_{\nu}$. Despite being Yukawa suppressed, we find that requiring $\delta m_{\nu}<1 \mathrm{eV}$ sets a bound on $\alpha_{H N e}$ orders of magnitude stronger than the current bound based on limits from $W \rightarrow \ell \nu$.

\section{Acknowledgments}

We would like to thank Claudius Krause and Jose Santiago for useful discussions. MC is supported by the Spanish MINECO under the Juan de la Cierva programme as well as by the Ministry of Science and Innovation under grant number FPA2016-78220-C3-3-P, and by the Junta de Andalucía grants FQM 101 and A-FQM-211-UGR18 (fondos FEDER).

\section{A Cross-checks}

Our partial yet thorough cross-check consists in computing the gauge dependence of UV divergences in the sector of $\alpha_{H N}, \alpha_{H N e}$ and $\alpha_{L N H}$, evaluating by hand (with the help of FeynRules [76]) each of the diagrams generated with QGRAF [77]. 


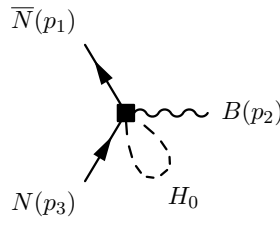

(1)

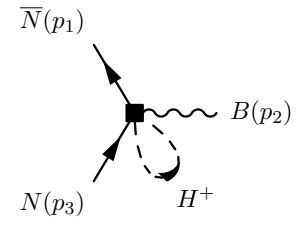

(2)

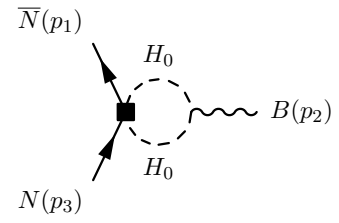

$(3)$

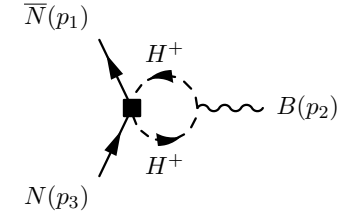

(4)

Figure 1. Relevant Feynman diagrams for $\bar{N} N \rightarrow B$.

We will make use of the following identities [42]:

$$
\begin{aligned}
& \int \frac{\mathrm{d}^{d} k}{(2 \pi)^{d}} \frac{1}{\left(k^{2}-M^{2}\right)^{n}}=A_{n}, \\
& \int \frac{\mathrm{d}^{d} k}{(2 \pi)^{d}} \frac{k^{\mu} k^{\nu}}{\left(k^{2}-M^{2}\right)^{n}}=g^{\mu \nu} B_{n}, \\
& \int \frac{\mathrm{d}^{d} k}{(2 \pi)^{d}} \frac{k^{\mu} k^{\nu} k^{\rho} k^{\sigma}}{\left(k^{2}-M^{2}\right)^{n}}=\left(g^{\mu \nu} g^{\rho \sigma}+g^{\mu \rho} g^{\nu \sigma}+g^{\mu \sigma} g^{\nu \rho}\right) C_{n},
\end{aligned}
$$

that lead to

$$
\begin{aligned}
A_{2} & =\frac{i}{16 \pi^{2} \epsilon}+\cdots, \\
B_{3} & =\frac{i}{64 \pi^{2} \epsilon}+\cdots, \\
C_{4} & =\frac{i}{384 \pi^{2} \epsilon}+\cdots,
\end{aligned}
$$

where the ellipses encode finite terms.

\section{A.1 $\bar{N} N \rightarrow B$}

The relevant diagrams are given in figure 1 . The different contributions to the amplitude read:

$$
\begin{aligned}
i \mathcal{M}_{1} & =g_{1} \alpha_{H N} A_{1} \overline{v_{1}} \gamma^{\mu} P_{R} u_{3} \epsilon_{\mu}^{*}, \\
\mathcal{M}_{2} & =\mathcal{M}_{1} \\
i \mathcal{M}_{3} & =-\frac{g_{1}}{2} \alpha_{H N} \overline{v_{1}}\left[4 B_{2} \gamma^{\mu}+\left(16 C_{4}-4 B_{3}\right) p_{2}^{2} \gamma^{\mu}+\left(A_{2}-8 B_{3}+32 C_{4}\right) p_{2}^{\mu} \not p_{2}\right] P_{R} u_{3} \epsilon_{\mu}^{*} \\
\mathcal{M}_{4} & =\mathcal{M}_{3} .
\end{aligned}
$$

Here and in what follows $v_{1} \equiv v\left(p_{1}\right), u_{3} \equiv u\left(p_{3}\right)$ and $\epsilon_{\mu}^{*} \equiv \epsilon_{\mu}^{*}\left(-p_{2}\right)$.

Summing over the four diagrams we obtain

$$
i \mathcal{M}_{\text {loop }}=\frac{i}{48 \pi^{2} \epsilon} g_{1} \alpha_{H N} \overline{v_{1}}\left(p_{2}^{2} \gamma^{\mu}-p_{2}^{\mu} \not p_{2}\right) P_{R} u_{3} \epsilon_{\mu}^{*} .
$$




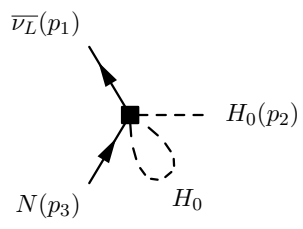

$(2)$

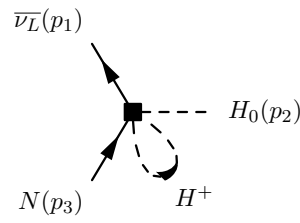

$(3)$

Figure 2. Relevant Feynman diagrams for $\overline{\nu_{L}} N \rightarrow H_{0}$.

The FeynArts/FormCalc convention for momenta differs from our setup involving QGRAF and FeynRules. In the former, the momenta of incoming particles point in and outgoing particles point out, while in the second all momenta point in. The momentum associated to each particle also differs.

Thus, we have $p_{3}^{\mathrm{FC}}=-p_{2}^{\mathrm{QG}}, p_{1}^{\mathrm{FC}}=p_{1}^{\mathrm{QG}}$ and $p_{2}^{\mathrm{FC}}=p_{3}^{\mathrm{QG}}$. Having this in mind, it is evident that this result agrees with eq. (3.1) in the limit $Y_{e}, \alpha_{N B}, \alpha_{N W} \rightarrow 0$.

\section{A.2 $\overline{\nu_{L}} N \rightarrow H_{0}$}

The relevant diagrams are shown in figure $2 .{ }^{6}$ We have:

$$
\begin{aligned}
& \mathcal{M}_{2}=0, \\
& \mathcal{M}_{3}=0 .
\end{aligned}
$$

Obviously, within our approximation this result agrees with eq. (3.5), which only depends on $\alpha_{N B}$ and $\alpha_{N W}$ (and on $\alpha_{H N e}$ through $Y_{e}$ ).

\section{$\mathrm{A.3} \quad \overline{\nu_{L}} N \rightarrow B H_{0}$}

The two relevant diagrams are those in figure 3. We obtain trivially

$$
\begin{aligned}
& \mathcal{M}_{1}=0, \\
& \mathcal{M}_{2}=0 .
\end{aligned}
$$

This again matches eq. (3.11) given our approximations.

\section{$\mathrm{A} .4 \quad \overline{e_{L}} N \rightarrow W^{3} H^{+}$}

In this case we have the two diagrams of figure 4, which lead to

$$
\begin{aligned}
& \mathcal{M}_{3}=0, \\
& \mathcal{M}_{4}=0,
\end{aligned}
$$

in agreement (within our approximation) with eq. (3.14).

\footnotetext{
${ }^{6}$ In what follows the "missing" diagrams are either of order $\mathcal{O}\left(\alpha_{N B, N W}\right)$ or $\mathcal{O}\left(1 / \Lambda^{4}\right)$. This is why we do not display them.
} 


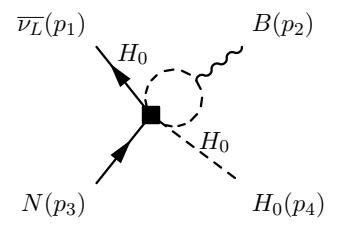

(1)

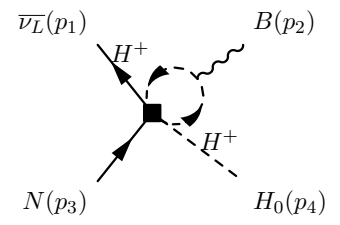

(2)

Figure 3. Relevant Feynman diagrams for $\overline{\nu_{L}} N \rightarrow B H_{0}$.

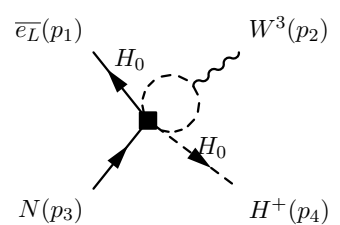

(3)

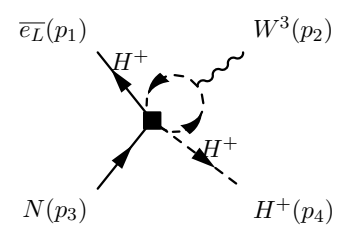

(4)

Figure 4. Relevant Feynman diagrams for $\overline{e_{L}} N \rightarrow W^{3} H^{+}$.

\section{A.5 $\bar{N} N \rightarrow H_{0}^{*} H_{0}$}

We have eleven relevant Feynman diagrams for this amplitude. They are depicted in figure 5. The different contributions read:

$$
\begin{aligned}
i \mathcal{M}_{1} & =g_{1}^{2}\left(A_{2}-B_{3}\right) \alpha_{H N} \overline{v_{1}} \not_{2} P_{R} u_{3}, \\
i \mathcal{M}_{2} & =g_{2}^{2}\left(A_{2}-B_{3}\right) \alpha_{H N} \overline{v_{1}} \not_{2} P_{R} u_{3}, \\
i \mathcal{M}_{3} & =g_{2}^{2}\left(A_{2}+2 B_{3}\right) \alpha_{H N} \overline{v_{1}} \not_{2} P_{R} u_{3}, \\
i \mathcal{M}_{4} & =-\frac{g_{1}^{2}}{2}\left(A_{2}+2 B_{3}\right) \alpha_{H N} \overline{v_{1}} \not_{4} P_{R} u_{3}, \\
i \mathcal{M}_{5} & =-\frac{g_{2}^{2}}{2}\left(A_{2}+2 B_{3}\right) \alpha_{H N} \overline{v_{1}} \not p_{4} P_{R} u_{3}, \\
i \mathcal{M}_{6} & =-g_{2}^{2}\left(A_{2}+2 B_{3}\right) \alpha_{H N} \overline{v_{1}} \not p_{4} P_{R} u_{3}, \\
i \mathcal{M}_{7} & =2 \lambda_{H}\left(A_{2}-4 B_{3}\right) \alpha_{H N} \overline{v_{1}}\left(\not p_{2}+\not p_{4}\right) P_{R} u_{3}, \\
i \mathcal{M}_{8} & =\lambda_{H}\left(A_{2}-4 B_{3}\right) \alpha_{H N} \overline{v_{1}}\left(\not p_{2}+\not p_{4}\right) P_{R} u_{3}, \\
i \mathcal{M}_{15} & =-\frac{g_{1}^{2}}{4} A_{2} \alpha_{H N} \overline{v_{1}}\left(\not p_{2}-\not p_{4}\right) P_{R} u_{3}, \\
i \mathcal{M}_{16} & =-\frac{g_{2}^{2}}{4} A_{2} \alpha_{H N} \overline{v_{1}}\left(\not p_{2}-\not p_{4}\right) P_{R} u_{3}, \\
i \mathcal{M}_{17} & =-\frac{g_{2}^{2}}{2} A_{2} \alpha_{H N} \overline{v_{1}}\left(\not p_{2}-\not p_{4}\right) P_{R} u_{3} .
\end{aligned}
$$

Summing over all them we arrive at

$$
i \mathcal{M}_{\text {loop }}=\frac{i}{32 \pi^{2} \epsilon}\left(g_{1}^{2}+3 g_{2}^{2}\right) \alpha_{H N} \overline{v_{1}}\left(\not p_{2}-\not p_{4}\right) P_{R} u_{3} .
$$




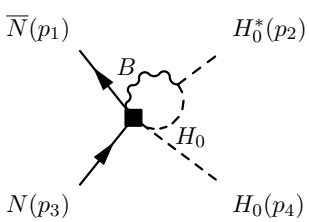

(1)

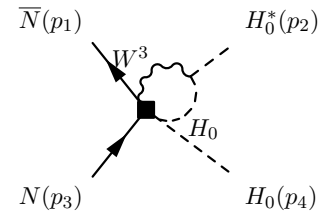

(2)

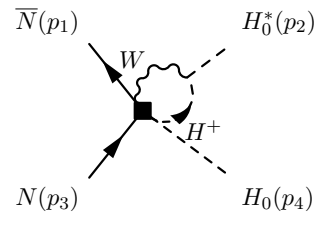

(3)

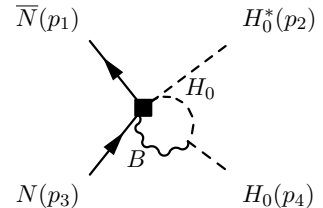

(4)

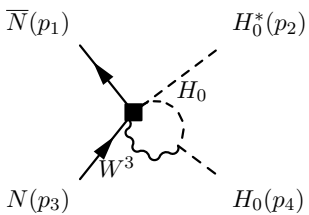

(5)

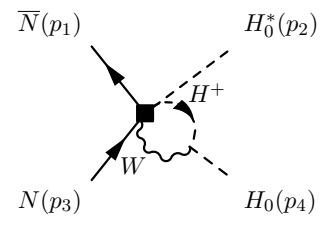

(6)

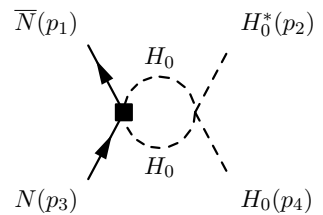

(7)

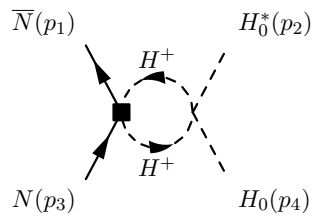

(8)

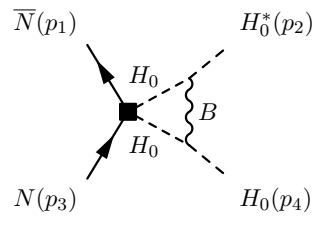

(15)

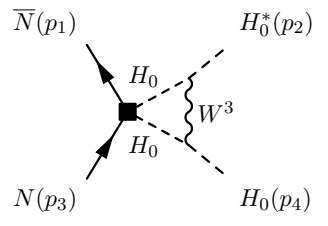

(16)

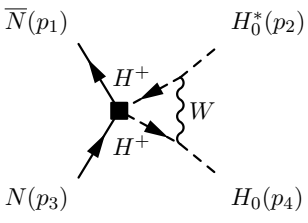

(17)

Figure 5. Relevant Feynman diagrams for $\bar{N} N \rightarrow H_{0}^{*} H_{0}$.

In this case, $p_{1}^{\mathrm{FC}}=p_{1}^{\mathrm{QG}}, p_{2}^{\mathrm{FC}}=p_{3}^{\mathrm{QG}}, p_{3}^{\mathrm{FC}}=-p_{2}^{\mathrm{QG}}$ and $p_{4}^{\mathrm{FC}}=-p_{4}^{\mathrm{QG}}$. Using this information, we match precisely eq. (3.17).

\section{A.6 $\overline{\nu_{L}} N H_{0}^{*} \rightarrow H_{0}^{*} H_{0}$}

21 diagrams need to be computed in this case. They are all shown in figure 6 . We have:

$$
\begin{aligned}
i \mathcal{M}_{9} & =4 \lambda_{H} A_{2} \alpha_{L N H} \overline{v_{1}} P_{R} u_{3}, \\
\mathcal{M}_{10} & =\frac{1}{4} \mathcal{M}_{9}, \\
\mathcal{M}_{11} & =\frac{1}{2} \mathcal{M}_{9}, \\
\mathcal{M}_{13} & =\mathcal{M}_{9}, \\
\mathcal{M}_{14} & =\frac{1}{4} \mathcal{M}_{9}, \\
i \mathcal{M}_{15} & =-\frac{g_{1}^{2}}{2} A_{2} \alpha_{L N H} \overline{v_{1}} P_{R} u_{3}, \\
i \mathcal{M}_{16} & =-\frac{g_{2}^{2}}{2} A_{2} \alpha_{L N H} \overline{v_{1}} P_{R} u_{3}, \\
\mathcal{M}_{17} & =\mathcal{M}_{16},
\end{aligned}
$$




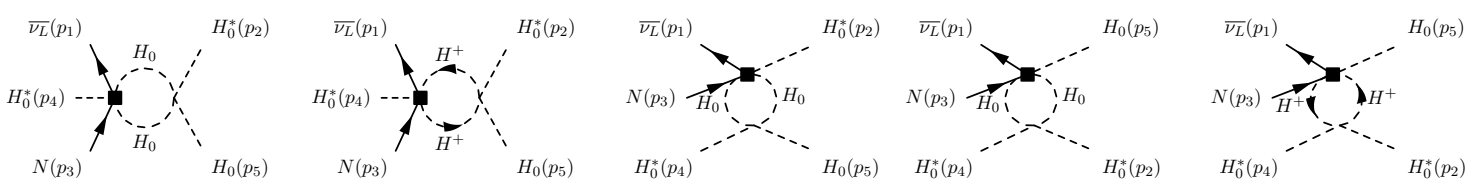

(9)

(10)

(11)

(13)

(14)

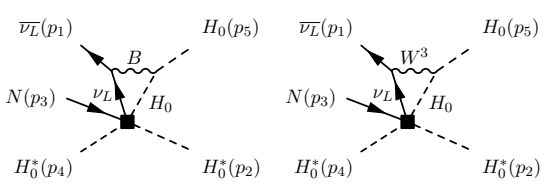

(15)

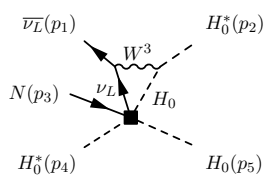

(19)

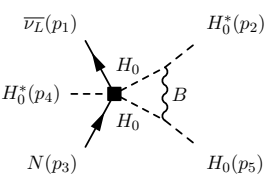

(24)

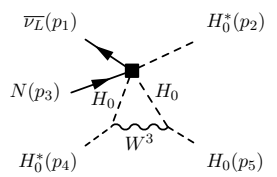

(28)
(16)

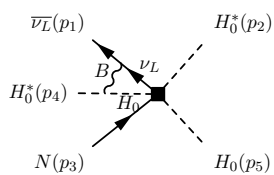

20

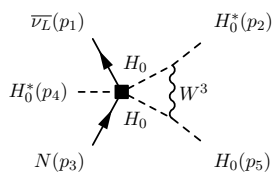

25

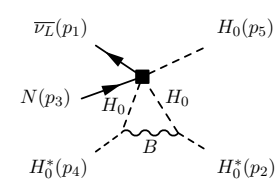

(30)

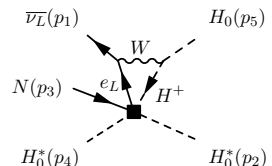

(17

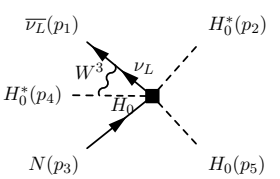

(21)

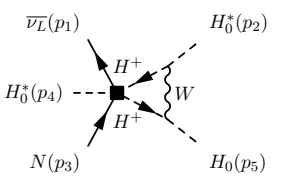

$(26)$

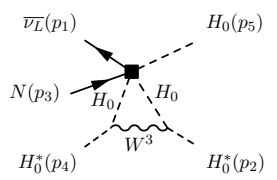

$(31)$

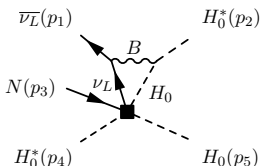

(18)

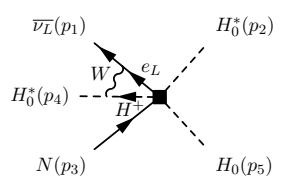

(22)

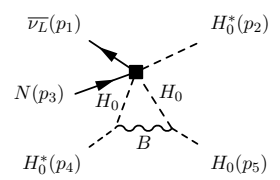

(27)

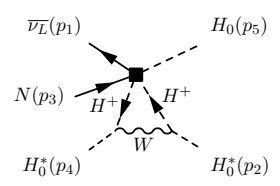

(32)

Figure 6. Relevant Feynman diagrams for $\overline{\nu_{L}} N H_{0}^{*} \rightarrow H_{0}^{*} H_{0}$.

$$
\begin{aligned}
& \mathcal{M}_{18}=-\mathcal{M}_{15}, \\
& \mathcal{M}_{19}=-\mathcal{M}_{16}, \\
& \mathcal{M}_{20}=\mathcal{M}_{15}, \\
& \mathcal{M}_{21}=\mathcal{M}_{16}, \\
& \mathcal{M}_{22}=\mathcal{M}_{16}, \\
& \mathcal{M}_{24}=\mathcal{M}_{15}, \\
& \mathcal{M}_{25}=\mathcal{M}_{16}, \\
& \mathcal{M}_{26}=\mathcal{M}_{16}, \\
& \mathcal{M}_{27}=-\mathcal{M}_{15}, \\
& \mathcal{M}_{28}=-\mathcal{M}_{16},
\end{aligned}
$$




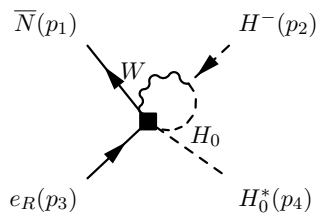

(1)

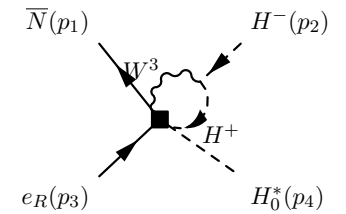

(2)

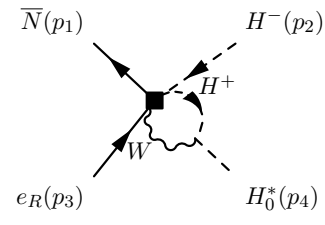

(3)

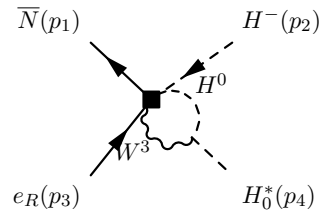

(4)

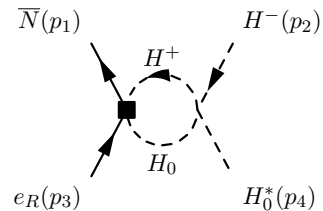

(5)

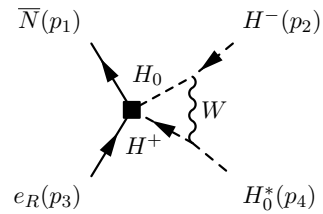

(10)

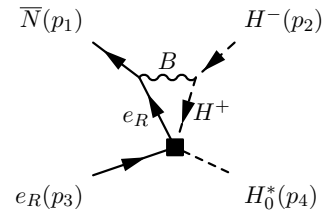

(8)

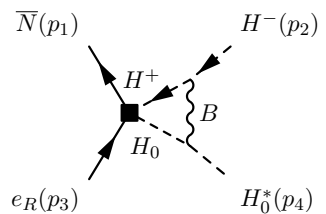

$(11)$

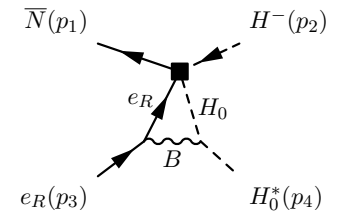

(9)

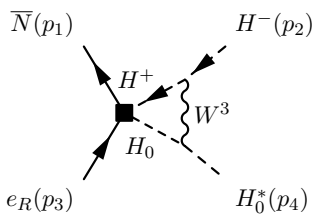

(12)

Figure 7. Relevant Feynman diagrams for $\bar{N} e_{R} \rightarrow H^{-} H_{0}^{*}$.

$$
\begin{aligned}
& \mathcal{M}_{30}=\mathcal{M}_{15}, \\
& \mathcal{M}_{31}=\mathcal{M}_{16}, \\
& \mathcal{M}_{32}=\mathcal{M}_{16} .
\end{aligned}
$$

As a result, we obtain

$$
i \mathcal{M}_{\text {loop }}=\frac{i}{16 \pi^{2} \epsilon}\left(12 \lambda_{H}-g_{1}^{2}-3 g_{2}^{2}\right) \alpha_{L N H} \overline{v_{1}} P_{R} u_{3},
$$

in agreement (within our approximation) with eq. (3.21).

\section{A.7 $\bar{N} e_{R} \rightarrow H^{-} H_{0}^{*}$}

Finally, this amplitude splits into the ten diagrams of figure 7 . We have

$$
\begin{aligned}
i \mathcal{M}_{1} & =2 g_{2}^{2}\left(A_{2}-B_{3}\right) \alpha_{H N e} \overline{v_{1}} \not p_{2} P_{R} u_{3}, \\
\mathcal{M}_{2} & =\frac{1}{2} \mathcal{M}_{1}, \\
i \mathcal{M}_{3} & =-2 g_{2}^{2}\left(A_{2}-B_{3}\right) \alpha_{H N e} \overline{v_{1}} \not p_{4} P_{R} u_{3}, \\
\mathcal{M}_{4} & =\frac{1}{2} \mathcal{M}_{3},
\end{aligned}
$$




$$
\begin{aligned}
i \mathcal{M}_{5} & =-\lambda_{H}\left(A_{2}-4 B_{3}\right) \alpha_{H N e} \overline{v_{1}}\left(\not p_{2}+\not p_{4}\right) P_{R} u_{3}, \\
i \mathcal{M}_{8} & =-g_{1}^{2} \alpha_{H N e} \overline{v_{1}}\left[\left(A_{2}+B_{3}\right) \not p_{2}+\frac{1}{2} A_{2}\left(\not p_{3}-\not p_{4}\right)\right] P_{R} u_{3}, \\
i \mathcal{M}_{9} & =g_{1}^{2} \alpha_{H N e} \overline{v_{1}}\left[\left(A_{2}+B_{3}\right) \not p_{4}+\frac{1}{2} A_{2}\left(\not p_{3}-\not p_{2}\right)\right] P_{R} u_{3}, \\
i \mathcal{M}_{10} & =-\frac{1}{2} g_{2}^{2} A_{2} \alpha_{H N e} \overline{v_{1}}\left(\not p_{2}-\not p_{4}\right) P_{R} u_{3}, \\
i \mathcal{M}_{11} & =\frac{1}{4} g_{1}^{2} A_{2} \alpha_{H N e} \overline{v_{1}}\left(\not p_{2}-\not p_{4}\right) P_{R} u_{3}, \\
\mathcal{M}_{12} & =\frac{1}{2} \mathcal{M}_{10} .
\end{aligned}
$$

Thus, we finally obtain:

$$
i \mathcal{M}_{\text {loop }}=-\frac{3 i}{32 \pi^{2} \epsilon}\left(g_{1}^{2}-g_{2}^{2}\right) \alpha_{H N e} \overline{v_{1}}\left(\not p_{2}-\not p_{4}\right) P_{R} u_{3}
$$

In this case, $p_{1}^{\mathrm{FC}}=p_{1}^{\mathrm{QG}}, p_{2}^{\mathrm{FC}}=p_{3}^{\mathrm{QG}}, p_{3}^{\mathrm{FC}}=-p_{2}^{\mathrm{QG}}$, and $p_{4}^{\mathrm{FC}}=-p_{4}^{\mathrm{QG}}$. So we easily see that we match eq. (3.24).

Open Access. This article is distributed under the terms of the Creative Commons Attribution License (CC-BY 4.0), which permits any use, distribution and reproduction in any medium, provided the original author(s) and source are credited.

\section{References}

[1] W. Buchmüller and D. Wyler, Effective Lagrangian Analysis of New Interactions and Flavor Conservation, Nucl. Phys. B 268 (1986) 621 [inSPIRE].

[2] B. Grzadkowski, M. Iskrzynski, M. Misiak and J. Rosiek, Dimension-Six Terms in the Standard Model Lagrangian, JHEP 10 (2010) 085 [arXiv: 1008.4884] [INSPIRE].

[3] I. Brivio and M. Trott, The Standard Model as an Effective Field Theory, Phys. Rept. 793 (2019) 1 [arXiv: 1706 . 08945] [INSPIRE].

[4] CMS collaboration, Angular analysis of the decay $B^{0} \rightarrow K^{* 0} \mu^{+} \mu^{-}$from pp collisions at $\sqrt{s}=8$ TeV, Phys. Lett. B 753 (2016) 424 [arXiv:1507.08126] [InSPIRE].

[5] CMS collaboration, Measurement of angular parameters from the decay $B^{0} \rightarrow K^{* 0} \mu^{+} \mu^{-}$in proton-proton collisions at $\sqrt{s}=8 \mathrm{TeV}$, Phys. Lett. B 781 (2018) 517 [arXiv:1710.02846] [INSPIRE].

[6] XENON collaboration, Observation of Excess Electronic Recoil Events in XENON1T, arXiv:2006.09721 [INSPIRE].

[7] C. Grojean, E.E. Jenkins, A.V. Manohar and M. Trott, Renormalization Group Scaling of Higgs Operators and $h \rightarrow \gamma \gamma$, JHEP 04 (2013) 016 [arXiv:1301.2588] [INSPIRE].

[8] J. Elias-Miró, J.R. Espinosa, E. Masso and A. Pomarol, Renormalization of dimension-six operators relevant for the Higgs decays $h \rightarrow \gamma \gamma, \gamma Z$, JHEP 08 (2013) 033 [arXiv:1302.5661] [INSPIRE]. 
[9] J. Elias-Miró, J.R. Espinosa, E. Masso and A. Pomarol, Higgs windows to new physics through $d=6$ operators: constraints and one-loop anomalous dimensions, JHEP 11 (2013) 066 [arXiv:1308.1879] [INSPIRE].

[10] E.E. Jenkins, A.V. Manohar and M. Trott, Renormalization Group Evolution of the Standard Model Dimension Six Operators I: Formalism and lambda Dependence, JHEP 10 (2013) 087 [arXiv: 1308.2627] [INSPIRE].

[11] E.E. Jenkins, A.V. Manohar and M. Trott, Renormalization Group Evolution of the Standard Model Dimension Six Operators II: Yukawa Dependence, JHEP 01 (2014) 035 [arXiv: 1310.4838$]$ [INSPIRE].

[12] R. Alonso, E.E. Jenkins, A.V. Manohar and M. Trott, Renormalization Group Evolution of the Standard Model Dimension Six Operators III: Gauge Coupling Dependence and Phenomenology, JHEP 04 (2014) 159 [arXiv:1312.2014] [INSPIRE].

[13] Y. Liao and X.-D. Ma, Renormalization Group Evolution of Dimension-seven Operators in Standard Model Effective Field Theory and Relevant Phenomenology, JHEP 03 (2019) 179 [arXiv: 1901.10302] [INSPIRE].

[14] E.E. Jenkins, A.V. Manohar and P. Stoffer, Low-Energy Effective Field Theory below the Electroweak Scale: Anomalous Dimensions, JHEP 01 (2018) 084 [arXiv:1711.05270] [INSPIRE].

[15] E.E. Jenkins, A.V. Manohar and P. Stoffer, Low-Energy Effective Field Theory below the Electroweak Scale: Operators and Matching, JHEP 03 (2018) 016 [arXiv:1709.04486] [INSPIRE].

[16] W. Dekens and P. Stoffer, Low-energy effective field theory below the electroweak scale: matching at one loop, JHEP 10 (2019) 197 [arXiv:1908.05295] [INSPIRE].

[17] B. Henning, X. Lu and H. Murayama, How to use the Standard Model effective field theory, JHEP 01 (2016) 023 [arXiv: 1412.1837] [INSPIRE].

[18] B. Henning, X. Lu and H. Murayama, One-loop Matching and Running with Covariant Derivative Expansion, JHEP 01 (2018) 123 [arXiv:1604.01019] [INSPIRE].

[19] S.A.R. Ellis, J. Quevillon, T. You and Z. Zhang, Mixed heavy-light matching in the Universal One-Loop Effective Action, Phys. Lett. B 762 (2016) 166 [arXiv:1604.02445] [INSPIRE].

[20] J. Fuentes-Martin, J. Portoles and P. Ruiz-Femenia, Integrating out heavy particles with functional methods: a simplified framework, JHEP 09 (2016) 156 [arXiv:1607.02142] [INSPIRE].

[21] Z. Zhang, Covariant diagrams for one-loop matching, JHEP 05 (2017) 152 [arXiv: 1610.00710] [INSPIRE].

[22] S.A.R. Ellis, J. Quevillon, T. You and Z. Zhang, Extending the Universal One-Loop Effective Action: Heavy-Light Coefficients, JHEP 08 (2017) 054 [arXiv: 1706. 07765] [INSPIRE].

[23] F. del Aguila, Z. Kunszt and J. Santiago, One-loop effective lagrangians after matching, Eur. Phys. J. C 76 (2016) 244 [arXiv:1602.00126] [INSPIRE].

[24] J.C. Criado, MatchingTools: a Python library for symbolic effective field theory calculations, Comput. Phys. Commun. 227 (2018) 42 [arXiv:1710.06445] [INSPIRE].

[25] S. Das Bakshi, J. Chakrabortty and S.K. Patra, CoDEx: Wilson coefficient calculator connecting SMEFT to UV theory, Eur. Phys. J. C 79 (2019) 21 [arXiv:1808.04403] [INSPIRE]. 
[26] J. Aebischer, M. Fael, A. Lenz, M. Spannowsky and J. Virto eds., Computing Tools for the SMEFT, based on the proceedings of the 1st Workshop on Tools for Low-Energy SMEFT Phenomenology (SMEFT-Tools 2019), Durham, United Kingdom, 12-14 June 2019, arXiv: 1910.11003 [INSPIRE].

[27] V. Gherardi, D. Marzocca and E. Venturini, Matching scalar leptoquarks to the SMEFT at one loop, JHEP 07 (2020) 225 [arXiv:2003.12525] [INSPIRE].

[28] L.E. Ibáñez, V. Martin-Lozano and I. Valenzuela, Constraining Neutrino Masses, the Cosmological Constant and BSM Physics from the Weak Gravity Conjecture, JHEP 11 (2017) 066 [arXiv: 1706.05392] [INSPIRE].

[29] N. Arkani-Hamed, S. Dubovsky, A. Nicolis and G. Villadoro, Quantum Horizons of the Standard Model Landscape, JHEP 06 (2007) 078 [hep-th/0703067] [INSPIRE].

[30] H. Ooguri and C. Vafa, Non-supersymmetric AdS and the Swampland, Adv. Theor. Math. Phys. 21 (2017) 1787 [arXiv:1610.01533] [INSPIRE].

[31] F. del Aguila, S. Bar-Shalom, A. Soni and J. Wudka, Heavy Majorana Neutrinos in the Effective Lagrangian Description: Application to Hadron Colliders, Phys. Lett. B 670 (2009) 399 [arXiv:0806.0876] [INSPIRE].

[32] Y. Liao and X.-D. Ma, Operators up to Dimension Seven in Standard Model Effective Field Theory Extended with Sterile Neutrinos, Phys. Rev. D 96 (2017) 015012 [arXiv: 1612.04527] [INSPIRE].

[33] L. Duarte, J. Peressutti and O.A. Sampayo, Majorana neutrino decay in an Effective Approach, Phys. Rev. D 92 (2015) 093002 [arXiv:1508.01588] [InSPIRE].

[34] L. Duarte, J. Peressutti and O.A. Sampayo, Not-that-heavy Majorana neutrino signals at the LHC, J. Phys. G 45 (2018) 025001 [arXiv: 1610.03894] [INSPIRE].

[35] J. Alcaide, S. Banerjee, M. Chala and A. Titov, Probes of the Standard Model effective field theory extended with a right-handed neutrino, JHEP 08 (2019) 031 [arXiv:1905.11375] [INSPIRE].

[36] J.M. Butterworth, M. Chala, C. Englert, M. Spannowsky and A. Titov, Higgs phenomenology as a probe of sterile neutrinos, Phys. Rev. D 100 (2019) 115019 [arXiv: 1909.04665] [INSPIRE].

[37] Y. Cai, T. Han, T. Li and R. Ruiz, Lepton Number Violation: Seesaw Models and Their Collider Tests, Front. Phys. 6 (2018) 40 [arXiv:1711.02180] [InSPIRE].

[38] I. Bischer and W. Rodejohann, General neutrino interactions from an effective field theory perspective, Nucl. Phys. B 947 (2019) 114746 [arXiv: 1905.08699] [InSPIRE].

[39] W. Dekens, J. de Vries, K. Fuyuto, E. Mereghetti and G. Zhou, Sterile neutrinos and neutrinoless double beta decay in effective field theory, JHEP 06 (2020) 097 [arXiv: 2002.07182] [INSPIRE].

[40] P.D. Bolton, F.F. Deppisch and C. Hati, Probing new physics with long-range neutrino interactions: an effective field theory approach, JHEP 07 (2020) 013 [arXiv: 2004.08328] [INSPIRE].

[41] L. Duarte, G. Zapata and O.A. Sampayo, Angular and polarization observables for Majorana-mediated B decays with effective interactions, arXiv:2006.11216 [INSPIRE].

[42] M. Chala and A. Titov, One-loop matching in the SMEFT extended with a sterile neutrino, JHEP 05 (2020) 139 [arXiv: 2001.07732] [INSPIRE]. 
[43] N.F. Bell, V. Cirigliano, M.J. Ramsey-Musolf, P. Vogel and M.B. Wise, How magnetic is the Dirac neutrino?, Phys. Rev. Lett. 95 (2005) 151802 [hep-ph/0504134] [INSPIRE].

[44] T. Han, J. Liao, H. Liu and D. Marfatia, Scalar and tensor neutrino interactions, JHEP 07 (2020) 207 [arXiv: 2004.13869] [inSPIRE].

[45] T. Hahn, Generating Feynman diagrams and amplitudes with FeynArts 3, Comput. Phys. Commun. 140 (2001) 418 [hep-ph/0012260] [INSPIRE].

[46] T. Hahn and M. Pérez-Victoria, Automatized one loop calculations in four-dimensions and D-dimensions, Comput. Phys. Commun. 118 (1999) 153 [hep-ph/9807565] [InSPIRE].

[47] G. Buchalla, A. Celis, C. Krause and J.-N. Toelstede, Master Formula for One-Loop Renormalization of Bosonic SMEFT Operators, arXiv:1904.07840 [INSPIRE].

[48] F. Takahashi, M. Yamada and W. Yin, XENON1T anomaly from anomaly-free ALP dark matter and its implications for stellar cooling anomaly, arXiv:2006.10035 [INSPIRE].

[49] K. Kannike, M. Raidal, H. Veermäe, A. Strumia and D. Teresi, Dark Matter and the XENON1T electron recoil excess, arXiv:2006.10735 [INSPIRE].

[50] G. Alonso-Álvarez, F. Ertas, J. Jaeckel, F. Kahlhoefer and L.J. Thormaehlen, Hidden Photon Dark Matter in the Light of XENON1T and Stellar Cooling, arXiv:2006.11243 [INSPIRE].

[51] B. Fornal, P. Sandick, J. Shu, M. Su and Y. Zhao, Boosted Dark Matter Interpretation of the XENON1T Excess, arXiv:2006.11264 [INSPIRE].

[52] C. Boehm, D.G. Cerdeno, M. Fairbairn, P.A.N. Machado and A.C. Vincent, Light new physics in XENON1T, arXiv:2006.11250 [INSPIRE].

[53] K. Harigaya, Y. Nakai and M. Suzuki, Inelastic Dark Matter Electron Scattering and the XENON1T Excess, Phys. Lett. B 809 (2020) 135729 [arXiv: 2006.11938] [InSPIRE].

[54] A. Bally, S. Jana and A. Trautner, Neutrino self-interactions and XENON1T electron recoil excess, arXiv:2006.11919 [INSPIRE].

[55] L. Su, W. Wang, L. Wu, J.M. Yang and B. Zhu, Atmospheric Dark Matter from Inelastic Cosmic Ray Collision in Xenon1T, arXiv:2006.11837 [INSPIRE].

[56] M. Du, J. Liang, Z. Liu, V.Q. Tran and Y. Xue, On-shell mediator dark matter models and the Xenon1T anomaly, arXiv:2006.11949 [INSPIRE].

[57] L. Di Luzio, M. Fedele, M. Giannotti, F. Mescia and E. Nardi, Solar axions cannot explain the XENON1T excess, arXiv:2006.12487 [INSPIRE].

[58] U.K. Dey, T.N. Maity and T.S. Ray, Prospects of Migdal Effect in the Explanation of XENON1T Electron Recoil Excess, arXiv:2006.12529 [INSPIRE].

[59] N.F. Bell, J.B. Dent, B. Dutta, S. Ghosh, J. Kumar and J.L. Newstead, Explaining the XENON1T excess with Luminous Dark Matter, arXiv:2006.12461 [INSPIRE].

[60] Y. Chen, J. Shu, X. Xue, G. Yuan and Q. Yuan, Sun Heated MeV-scale Dark Matter and the XENON1T Electron Recoil Excess, arXiv:2006.12447 [INSPIRE].

[61] D. Aristizabal Sierra, V. De Romeri, L.J. Flores and D.K. Papoulias, Light vector mediators facing XENON1T data, Phys. Lett. B 809 (2020) 135681 [arXiv:2006.12457] [InSPIRE].

[62] J. Buch, M.A. Buen-Abad, J. Fan and J.S.C. Leung, Galactic Origin of Relativistic Bosons and XENON1T Excess, arXiv:2006.12488 [INSPIRE].

[63] G. Choi, M. Suzuki and T.T. Yanagida, XENON1T Anomaly and its Implication for Decaying Warm Dark Matter, arXiv:2006.12348 [InSPIRE]. 
[64] G. Paz, A.A. Petrov, M. Tammaro and J. Zupan, Shining dark matter in Xenon1T, arXiv:2006.12462 [INSPIRE].

[65] H.M. Lee, Exothermic Dark Matter for XENON1T Excess, arXiv:2006.13183 [INSPIRE].

[66] R. Primulando, J. Julio and P. Uttayarat, Collider Constraints on a Dark Matter Interpretation of the XENON1T Excess, arXiv:2006.13161 [INSPIRE].

[67] K. Nakayama and Y. Tang, Gravitational Production of Hidden Photon Dark Matter in light of the XENON1T Excess, arXiv:2006.13159 [INSPIRE].

[68] Y. Jho, J.-C. Park, S.C. Park and P.-Y. Tseng, Leptonic New Force and Cosmic-ray Boosted Dark Matter for the XENON1T Excess, arXiv:2006.13910 [INSPIRE].

[69] M. Baryakhtar, A. Berlin, H. Liu and N. Weiner, Electromagnetic Signals of Inelastic Dark Matter Scattering, arXiv:2006.13918 [INSPIRE].

[70] H. An, M. Pospelov, J. Pradler and A. Ritz, New limits on dark photons from solar emission and keV scale dark matter, arXiv:2006.13929 [INSPIRE].

[71] A.E. Robinson, XENON1T observes tritium, arXiv: 2006.13278 [INSPIRE].

[72] A.N. Khan, Nonstandard Neutrino Interactions can explain the XENON1T spectral excess?, arXiv: 2006.12887 [INSPIRE].

[73] C. Giunti and A. Studenikin, Neutrino electromagnetic interactions: a window to new physics, Rev. Mod. Phys. 87 (2015) 531 [arXiv: 1403.6344] [INSPIRE].

[74] Particle Data Group, Review of Particle Physics, Phys. Rev. D 98 (2018) 030001 [INSPIRE].

[75] A. Ishikawa, Search for invisible decays of the Higgs boson at the ILC, PoS LeptonPhoton2019 (2019) 147 [arXiv:1909.07537] [INSPIRE].

[76] A. Alloul, N.D. Christensen, C. Degrande, C. Duhr and B. Fuks, FeynRules 2.0 - A complete toolbox for tree-level phenomenology, Comput. Phys. Commun. 185 (2014) 2250 [arXiv: 1310.1921] [INSPIRE].

[77] P. Nogueira, Automatic Feynman graph generation, J. Comput. Phys. 105 (1993) 279 [INSPIRE]. 